\title{
Reología de emulsiones estabilizadas por biopolímeros
}

\author{
Por J. M. Madiedo1, C. Bower2, M. R. Mackley² y C. Gallegos' \\ 1 Departamento de Ingeniería Química. Universidad de Sevilla. C/. P. García González, s/n. 41012 Sevilla (España) \\ 2 Department of Chemical Engineering. Cambridge University. Pembroke Street, Cambridge CB2 3RA (U.K.)
}

\section{RESUMEN}

\section{Reología de emulsiones estabilizadas por biopolímeros}

En este trabajo se estudian las respuestas viscosa y viscoelástica de emulsiones alimentarias estabilizadas por proteínas. La función de amortiguación determinada desde el módulo de relajación ha permitido, junto con el espectro de relajación calculado a partir de los módulos dinámicos, predecir la respuesta del material en condiciones de cizalla estacionaria mediante la aplicación del modelo de Wagner. Por otra parte, el estudio de la evolución de la microestructura de estos sistemas con la cizalla permite explicar el comportamiento observado en ensayos de flujo estacionario, asi como la falta de concordancia entre los resultados experimentales y los predichos.

PALABRAS-CLAVE: Biopolímero - Emulsión - Espectro Viscoelasticidad.

\section{SUMMARY}

\section{Rheology of biopolymer stabilized emulsions}

In this work, the viscous and viscoelastic responses of food emulsions stabilized by proteins are studied. The damping function determined from the relaxation modulus, together with the relaxation spectrum calculated from the dynamic moduli, allowed a prediction of the material response under steady-state conditions by applying the Wagner model. On the other hand, the study of the evolution of the microstructure of these systems under shear allows to explain the behavior observed in steady-flow tests, as well as the lack of concordance between experimental an predicted values.

KEY-WORDS: Biopolymer - Emulsion - SpectrumViscoelasticity.

\section{INTRODUCCIÓN}

La mayoría de las emulsiones alimentarias aceite en agua son estabilizadas por emulsionantes macromoleculares (biopolímeros). Así, las moléculas de proteína forman una barrera estérica, de protección contra la coalescencia, alrededor de las gotas de aceite.

La estabilidad de una emulsión alimentaria es el factor más importante a considerar en relación con sus aplicaciones industriales. La estabilidad de una emulsión depende de la distribución de tamaños de gotas de la fase dispersa, de la reología de la fase continua y de las interacciones entre partículas de fase dispersa (Melik et al., 1988). La floculación de las gotas de aceite en una emulsión en agua ha sido considerada tradicionalmente como un mecanismo de inestabilidad, ya que puede ser un paso intermedio hacia la coalescencia o favorecer el proceso de cremado debido a que la velocidad de sedimentación de los agregados es superior a la de las gotas individuales. Sin embargo, en emulsiones concentradas, un proceso generalizado de floculación puede favorecer la estabilidad de la emulsión al formarse un entramado tridimensional (Dickinson, 1989). La naturaleza y concentración del emulsionante usado es clave en este proceso de floculación.

La respuesta viscosa y viscoelástica de las emulsiones floculadas es muy compleja, y claramente distinta de la encontrada en emulsiones no floculadas (Tadros, 1990; Tadros, 1993). Así, medidas de cizalla oscilatoria llevadas a cabo con mayonesas (Berjano et al., 1990; Gallegos et al., 1992a) demostraron que la evolución de las funciones viscoelásticas lineales con la frecuencia presenta valores mínimos del módulo de pérdidas a frecuencias intermedias y una región «plateau» en el módulo de almacenamiento (con valores de la pendiente próximos a 0,1 ). No obstante, es de resaltar que la dependencia con la frecuencia de ambos módulos es función de la concentración de fase dispersa, condiciones de procesado y naturaleza del emulsionante usado (Franco et al., 1995; Franco et al., 1997). Consecuentemente, una representación más generalizada de la respuesta en cizalla oscilatoria sería: una región pseudo-terminal a bajas frecuencias, una región "plateau» intermedia y el comienzo de la región de transición a altas frecuencias. Los espectros de relajación calculados a partir de las funciones viscoelásticas dinámicas, bien por métodos aproximados (Franco et al., 1995; Franco et al., 1997) o por técnicas de regularización (Madiedo, 1996; Madiedo et al., 1996), pueden ser satisfactoriamente definidos por el modelo BSW-CW (De Rosa et al., 1994). Este modelo predice una transición brusca 
entre la región «plateau» y la pseudo-terminal del espectro (Franco et al., 1995). Madiedo et al. (1997) han usado un modelo empírico que describe las tres regiones del espectro antes citadas y donde la transición entre dichas regiones es mucho más paulatina.

Las emulsiones floculadas no siguen la regla Cox-Merz. Las curvas de flujo en estado estacionario muestran, a partir de velocidades de cizalla del orden de $10^{-4} \mathrm{~s}^{-1}$, una región pseudoplástica, donde la viscosidad es aproximadamente proporcional a $\gamma^{-1}$. Este comportamiento puede explicarse por un proceso de defloculación de gotas de aceite, aunque otros mecanismos pueden desarrollarse a medida que aumènta la velocidad de cizalla. Un comportamiento similar muestran las suspensiones floculadas (Barnes, 1995).

En cuanto a las características del flujo transitorio de las emulsiones floculadas, en ensayos donde la emulsión se somete a una velocidad de cizalla constante, se constata la existencia de un máximo, o sobreesfuerzo, en las curvas de crecimiento del esfuerzo con el tiempo de cizalla; la magnitud de este depende de la velocidad de cizalla (Gallegos et al., 1988).

Se han realizado diversos intentos para modelizar el flujo transitorio y en estado estacionario de emulsiones aceite en agua usando modelos viscoelásticos no lineales. Así Mackley et al. (1994) usaron sin éxito el modelo de Wagner y un espectro discreto de relajación para predecir el flujo estacionario de diversos materiales, concretamente para el caso de una mayonesa comercial. Otros autores (Gallegos et al., 1992b; Gallegos et al., 1995) usaron un modelo de Wagner modificado, donde la función de amortiguación de Wagner fue sustituida por la de Soskey-Winter, para ajustar el flujo transitorio de distintas emulsiones comerciales y modelos, obteniendo relativamente buenos resultados para bajos valores de velocidad de cizalla, aunque los valores absolutos del esfuerzo transitorio fueron difícilmente predecibles.

Asumiendo que las características del flujo de emulsiones floculadas aceite en agua deben estar relacionadas con la evolución de la estructura con la cizalla, este trabajo trata de estudiar comparativamente las modificaciones microestructurales inducidas por la cizalla en una emulsión floculada, usando un dispositivo óptico, y la evolución de la viscosidad en estado estacionario con la velocidad de cizalla para dicha emulsión, con el objetivo central de aportar nuevos datos y clarificar la falta de concordancia entre los valores experimentales y los predichos a partir de la modelización viscoelástica no lineal.

\section{EXPERIMENTAL}

Se han estudiado dos emulsiones estabilizadas por distintos tipo de proteínas. Así, se preparó una emulsión modelo con un contenido del $60 \%$ en peso de aceite de girasol (Hijos de Ybarra, S. A., Sevilla, España) y un $4 \%$ de proteína vegetal (Systems Bioindustries, S. A., Barcelona, España). Del mismo modo se estudió una mayonesa comercial que utiliza en su formulación huevo completo como emulsionante.

La caracterización reológica de las emulsiones se llevó a cabo en un espectrómetro dinámico, modelo RDSII, de la firma Rheometric Scientific, Ltd. (Surrey, Inglaterra), usando un sistema sensor tipo placa-placa (diámetro: $5 \mathrm{~cm}$ ). En primer lugar se determinó la extensión del intervalo viscoelástico lineal llevando a cabo un barrido de deformación. A continuación se realizó un barrido de frecuencias para conocer los valores de las funciones viscoelásticas dinámicas en un intervalo comprendido entre $10^{-2}$ y $10^{2} \mathrm{rad} / \mathrm{s}$. Seguidamente se realizaron ensayos de relajación tras someter el material a deformaciones tanto en la región viscoelástica lineal como en la no lineal. Por último, se estudió el flujo en estado estacionario de las mismas, en un intervalo de velocidades de cizalla comprendido entre 0,1 y $300 \mathrm{~s}^{-1}$.

Además de la caracterización reológica, se efectuaron observaciones microscópicas de los efectos de la velocidad de cizalla sobre la microestructura de la emulsión. Para este fin se usó un dispositivo óptico, que incluye una celdilla donde la muestra está siendo cizallada entre dos discos de cuarzo, de la firma Linkam Scientific Instruments (Surrey, Inglaterra).

\section{MODELIZACIÓN VISCOELÁSTICA NO LINEAL}

El modelo de Wagner (Wagner, 1976) es una ecuación constitutiva dependiente de la deformación tipo K-BKZ (Larson, 1988). Considerando cizalla simple, y asumiendo que la función memoria puede ser expresada como el producto de una componente dependiente sólo del tiempo y de otra componente que sólo depende de la deformación, el modelo de Wagner se reduce a la ecuación:

$$
\sigma_{12}(t)=\int_{-\infty}^{t} \frac{d G\left(t-t^{\prime}\right)}{d t^{\prime}} h\left(\gamma_{12}\right) \gamma_{12}\left(t, t^{\prime}\right) d t^{\prime}
$$

donde $\mathrm{G}\left(\mathrm{t}-\mathrm{t}^{\prime}\right)$ es el módulo de relajación lineal y $\mathrm{h}\left(\gamma_{12}\right)$ es la función amortiguación:

$$
h\left(\gamma_{12}\right)=e^{-k \gamma\left(t, t^{\prime}\right)}
$$

y en la que el factor de amortiguación, k, es un parámetro empírico que cuantifica el nivel de no linealidad en el material. Esta función se calcula a partir de la 
relación entre el módulo de relajación no lineal y el módulo de relajación lineal:

$$
h\left(\gamma_{12}\right)=\frac{G\left(\gamma, t-t^{\prime}\right)}{G\left(t-t^{\prime}\right)}
$$

El módulo de relajación lineal se relaciona con el espectro continuo de relajación según la ecuación:

$$
G\left(t-t^{\prime}\right)=\int_{-\infty}^{\infty} H(\lambda) e^{-\left(t-t^{\prime}\right) \lambda} d \ln \lambda
$$

Este espectro de relajación ha sido calculado usando técnicas de regularización (10), a partir de las funciones viscoelásticas dinámicas.

Sustituyendo la ecuación /4/ en la /1/, resulta:

$$
\sigma_{12}(t)=\int_{-\infty}^{t} \int_{-\infty}^{\infty} H(\lambda) e^{-\left(t-t^{\prime}\right) \lambda} e^{-k \gamma_{12}\left(t, t^{\prime}\right)} \gamma_{12}\left(t, t^{\prime}\right) d \ln \lambda d t^{\prime}
$$

y la viscosidad en estado estacionario resultante:

$$
\eta(\gamma)=\int_{-\infty}^{\infty} \frac{\lambda H(\lambda)}{(1+k \lambda \gamma)^{2}} d \ln \lambda
$$

\section{RESULTADOS Y DISCUSIÓN}

\section{Emulsión estabilizada por proteína vegetal}

Los datos reológicos de la emulsión estudiada preparada con una proteína vegetal como emulsionante se presentan en la figura 1. El barrido de deformación demuestra que la respuesta del material es lineal sólo en un intervalo de deformación muy limitado. Por ello, se realizó un barrido de frecuencia para una deformación del $0,4 \%$. La evolución de las funciones viscoelásticas con la frecuencia se presentan en la figura 1a, así como su recálculo a partir del espectro de relajación continuo (figura 1b) estimado a partir de técnicas de regularización. Como se ha dicho previamente, la dependencia de las funciones viscoelásticas con la frecuencia es típica de las emulsiones floculadas, con el módulo elástico superior al viscoso en todo el intervalo de frecuencias estudiado, lo que indica el comportamiento predominantemente elástico de la emulsión. El espectro de relajación muestra únicamente dos zonas diferenciadas, la de transición y la «plateau", esta última con una pendiente muy cercana a cero.

Los resultados de los ensayos de relajación se presentan en la figura 1c. Puede observarse una marcada respuesta no lineal, ya a deformaciones del $10 \%$, con acusados descensos del módulo de relajación en relación con los valores lineales de éste, obtenidos a deformaciones del orden del 1\% y comproba-
$\mathrm{G}^{\prime}, \mathrm{G}^{\prime \prime}(\mathrm{Pa})$

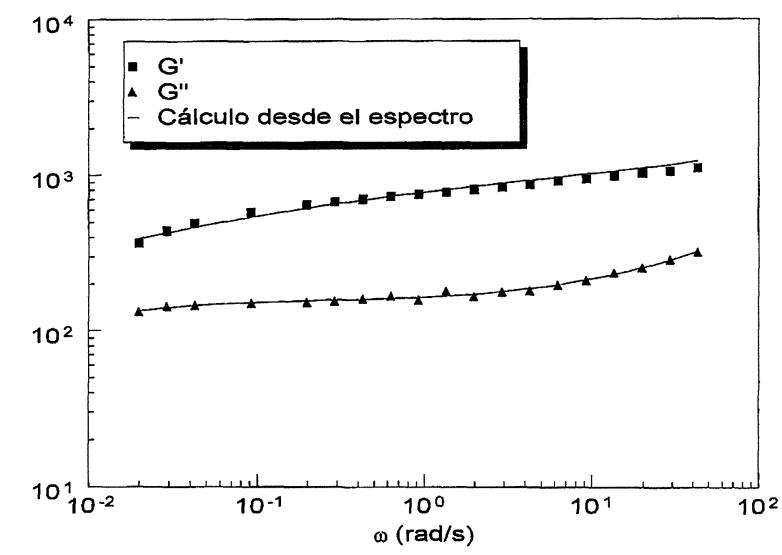

Figura 1a

Valores experimentales y recalculados de los módulos de almacenamiento y pérdidas para la emulsión estabilizada con proteína vegetal, a $25^{\circ} \mathrm{C}$.

$\mathrm{H}(\mathrm{Pa})$

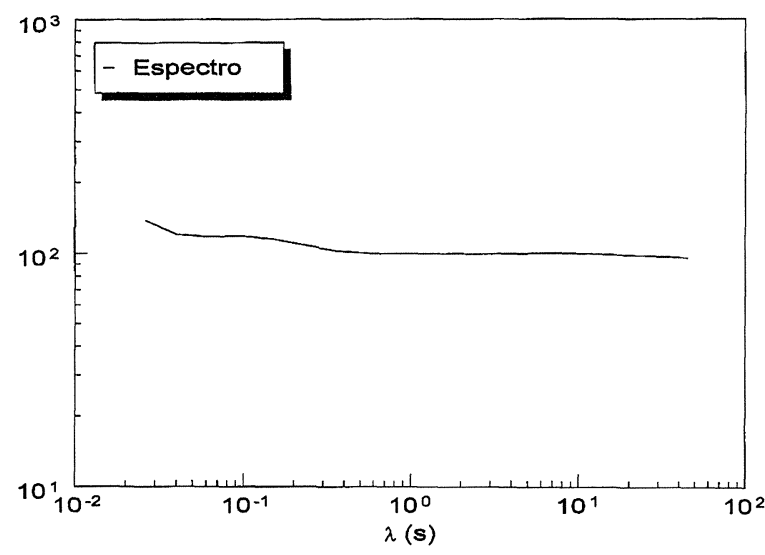

Figura 1b

Espectro de relajación para la emulsión estabilizada con proteína vegetal, a $25^{\circ} \mathrm{C}$, estimado a partir de las funciones dinámicas.

$G(t)(P a)$

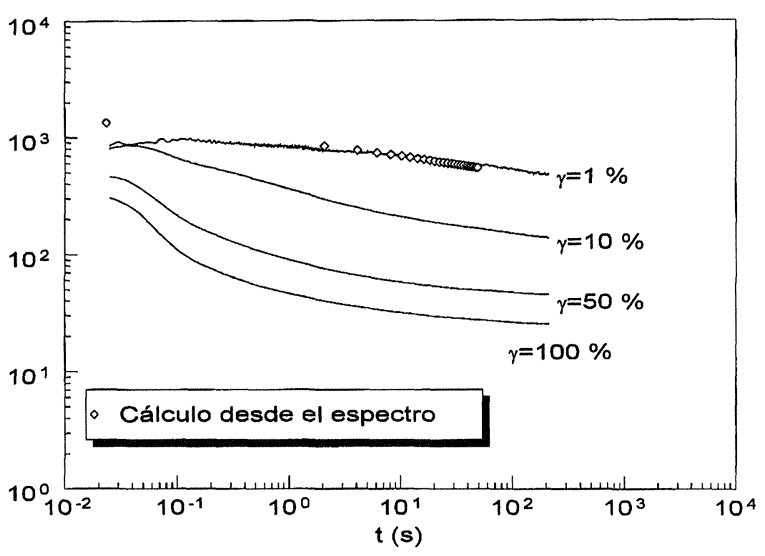

Figura 1c

Variación del módulo de cizalla con el tiempo a distintas deformaciones para la emulsión estabilizada mediante proteína vegetal, a $25^{\circ} \mathrm{C}$. 


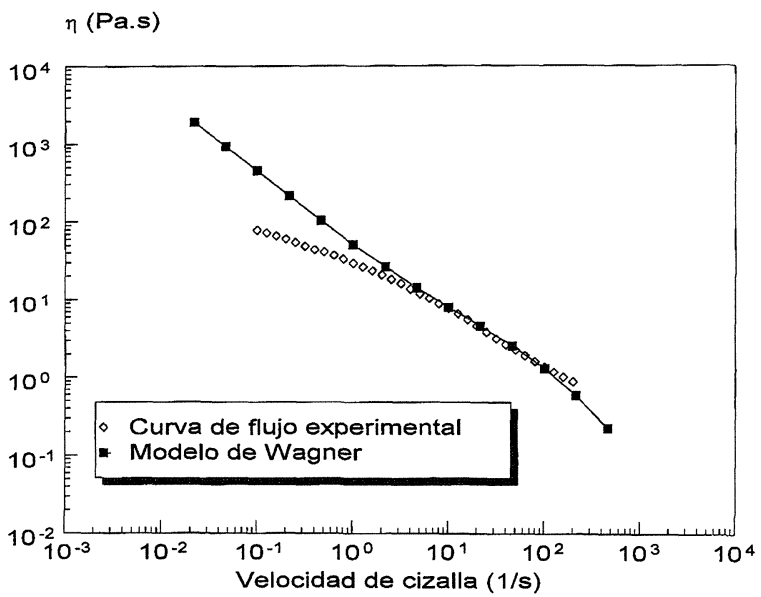

Figura 1d

Curva de flujo experimental y valores calculados a partir del modelo de Wagner para la emulsión estabilizada por proteína vegetal, a $25^{\circ} \mathrm{C}$

dos por su estimación a partir del espectro de relajación.

Estos datos han sido usados para calcular el factor de amortiguación de la función $h(\gamma)$, con el fin de predecir la respuesta del material sometido a cizalla en estado estacionario. Dicho factor, para la emulsión estudiada, tiene un valor de $\mathrm{k}=2,2$.

Por último, la figura 1d muestra la curva de flujo experimental en estado estacionario, a la temperatura de $25^{\circ} \mathrm{C}$, para la citada emulsión. En la misma figura se presenta la curva de flujo predicha a partir del modelo de Wagner, usando el factor de amortiguación antes citado y el espectro de relajación continuo.

Puede observarse que dicho modelo viscoelástico no lineal da una buena estimación de los resultados experimentales sobre dos décadas de velocidades de cizalla. La sobreestimación de la viscosidad aparente a bajas velocidades de cizalla puede atribuirse a efectos de deslizamiento en las paredes de las placas usadas (Barnes, 1995).

Esto fue confirmado por medio de observaciones experimentales de la microestructura de la emulsión sometida a cizalla. Así, sometida a bajas velocidades de cizalla $\left(\leq 10 s^{-1}\right)$ la muestra deslizaba entre las placas de cuarzo. Asimismo, se confirmó que se producían modificaciones en la distribución de tamaños de gotas de fase dispersa sólo a altas velocidades de cizalla, del orden de $1000 \mathrm{~s}^{-1}$.

\section{Emulsión estabilizada por proteínas de huevo (mayonesa comercial)}

Los resultados de los ensayos reológicos obtenidos con una mayonesa comercial que usa como emulsionante huevo completo se presentan en la figura 2.
En general, los datos son similares a los presentados anteriormente cuando se usó proteína vegetal como emulsionante. Así, la región viscoelástica lineal está limitada a deformaciones del orden del 1\%. El barrido de frecuencias realizado dentro de dicha región demuestra nuevamente que estas emulsiones son predominantemente elásticas, con valores del módulo de almacenamiento superiores a los del módulo de pérdidas en todo el intervalo de frecuencia estudiado (figura 2a). La figura $2 \mathrm{~b}$ muestra el espectro continuo de relajación de esta emulsión, calculado a partir de métodos de regularización. En este caso el perfil es casi totalmente plano, debido a que se dispone de pocos valores a altas frecuencias, donde debe empezar a aparecer la región de transición del citado espectro. Nuevamente el recálculo de las funciones viscoelásticas a partir de dicho espectro es totalmente satisfactorio.

\section{$\mathrm{G}^{\prime}, \mathrm{G} "(\mathrm{~Pa})$}

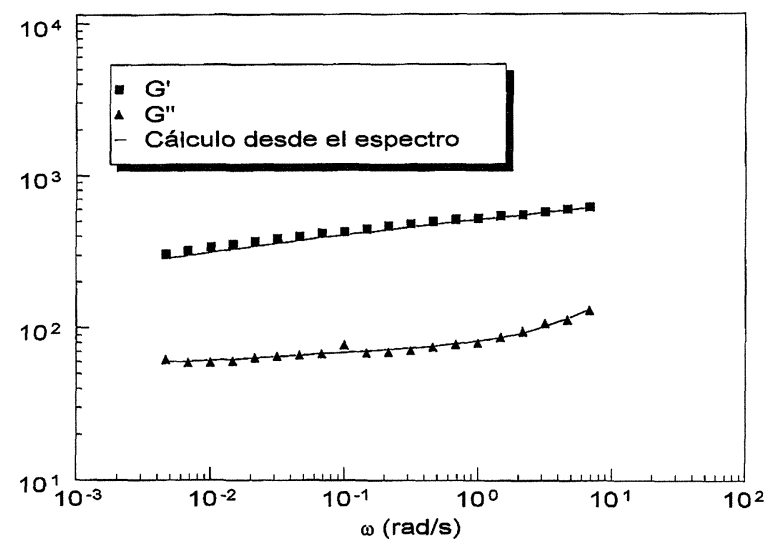

Figura $2 a$

Valores experimentales y recalculados de los módulos de almacenamiento y pérdidas para la mayonesa comercial, a $25^{\circ} \mathrm{C}$.

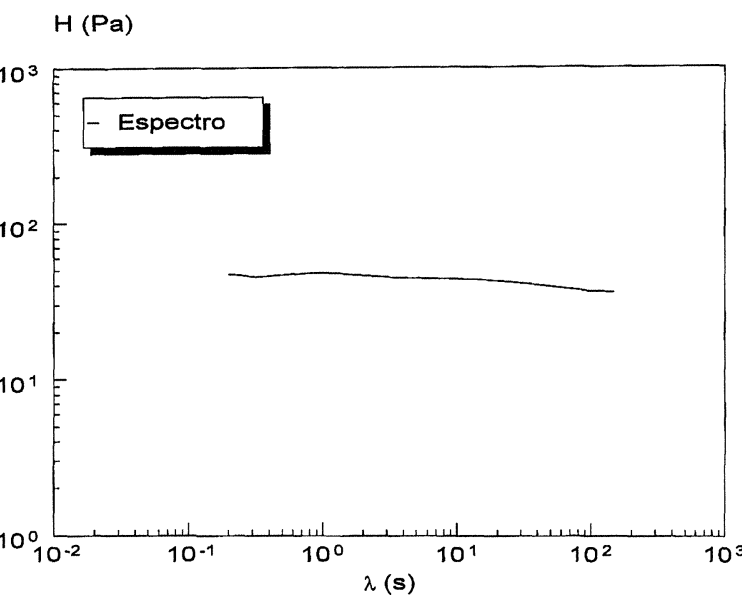

Figura $2 b$

Espectro de relajación para la mayonesa comercial, a $25^{\circ} \mathrm{C}$ estimado a partir de las funciones dinámicas. 
$\mathrm{G}(\mathrm{Pa})$

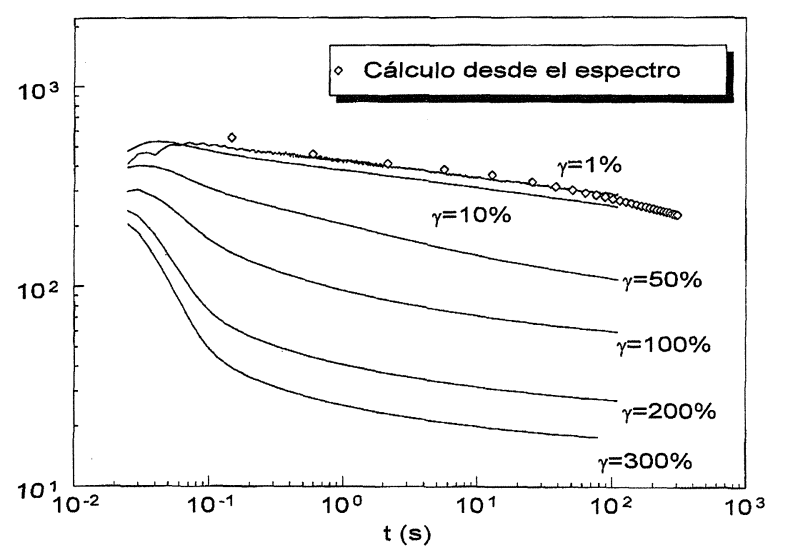

Figura 2c

Variación del módulo de cizalla con el tiempo a distintas deformaciones para la mayonesa comercial, a $25^{\circ} \mathrm{C}$.

Los ensayos de relajación del esfuerzo, en la región viscoelástica lineal y no lineal, realizados sobre la mayonesa comercial demuestran que ésta es más resistente a la destrucción del entramado tridimensional formado por la floculación extensiva de gotas de aceite que en el caso de la emulsión estabilizada con proteína vegetal, debido, entre otros factores, al mayor contenido en aceite de aquella. Así, una deformación del $10 \%$ produce un descenso menos importante en los valores del módulo de relajación (figura 2c). Consecuentemente, los valores del factor de amortiguación son significativamente menores en el caso de la mayonesa comercial $(k=0,75)$.

La figura $2 d$ muestra comparativamente la curva de flujo en estado estacionario experimental y la calculada a partir del modelo de Wagner para la mayonesa comercial. Como puede observarse, en este caso la concordancia entre ambas curvas es muy pobre en todo el intervalo de velocidades de cizalla, existiendo nuevamente una sobreestimación a bajas velocidades

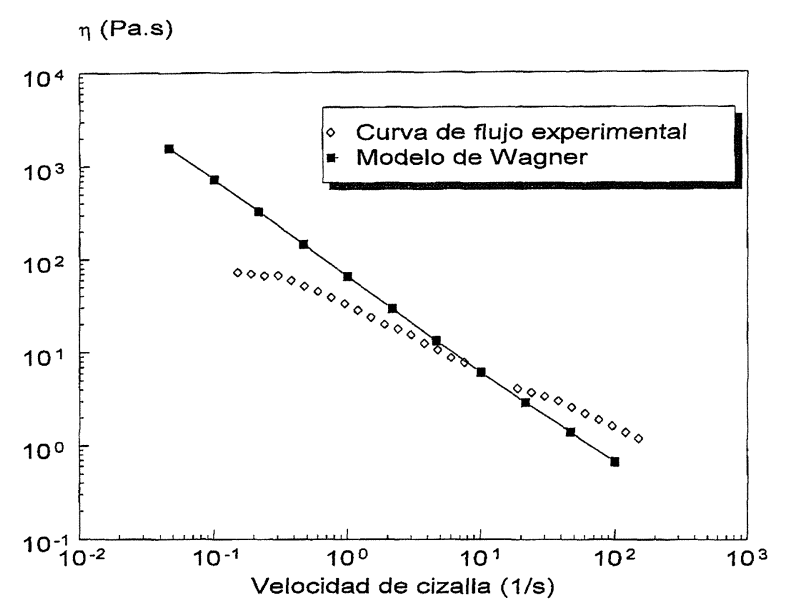

Figura 2d

Curva de flujo experimental y valores calculados a partir del modelo de Wagner para la mayonesa comercial, a $25^{\circ} \mathrm{C}$.

de cizalla, mientras que los valores experimentales son claramente superiores a los predichos a altas velocidades de cizalla.

Estos resultados pueden ser explicados por medio de las observaciones ópticas realizadas sobre muestras de mayonesas sometidas a velocidades de cizalla crecientes. Así, a bajas velocidades de cizalla las muestras presentaban efectos de deslizamiento entre las placas de cuarzo de la celda de cizalla, lo que explica que los valores experimentales sean inferiores a los predichos por el modelo de Wagner. Según aumenta la velocidad de cizalla desaparecen progresivamente los fenómenos de deslizamiento. Cuando se alcanzan velocidades de cizalla comprendida entre 100 y $300 \mathrm{~s}^{-1}$, se observa visualmente de forma muy clara que existe un proceso de coalescencia de gotas de aceite (figura 3). Una elevación posterior de la velocidad de cizalla conduce, de nuevo, a un proceso de rotura y a valores de distribución de tamaños de gota similares a los de la muestra no cizallada.

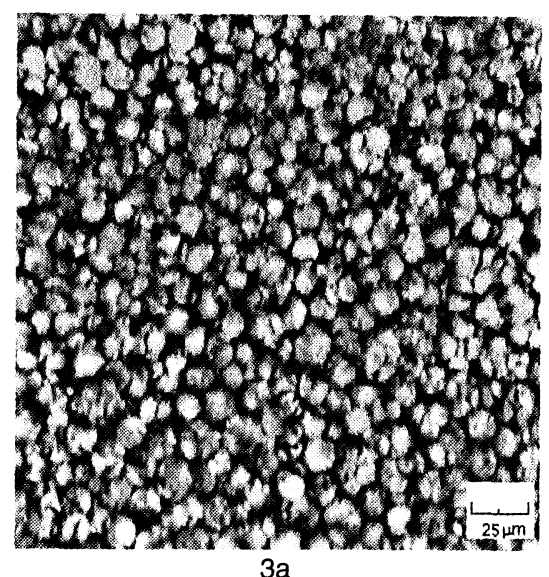

3a

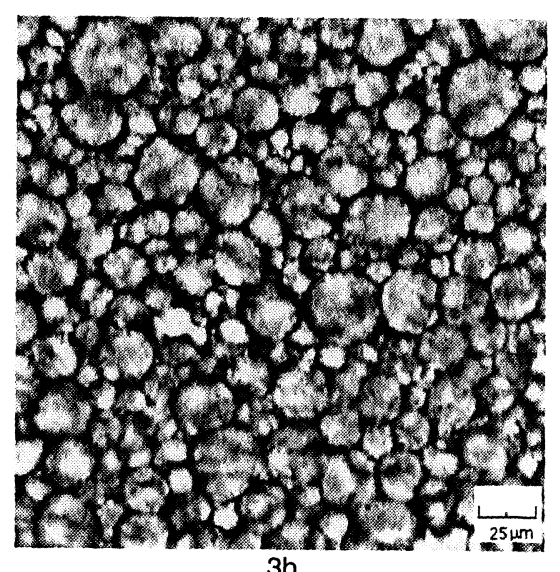

Figura 3.

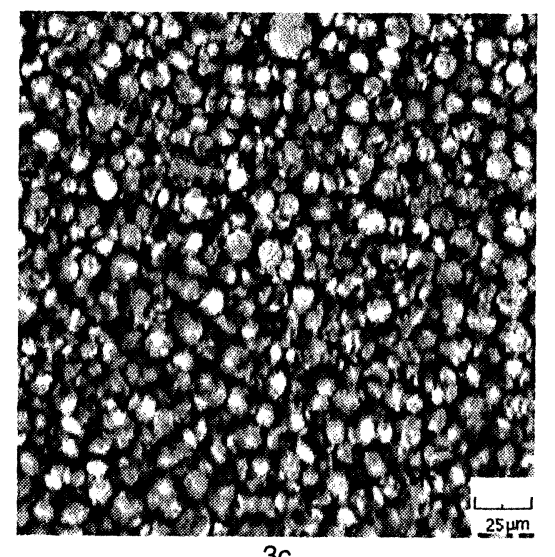

$3 c$

Influencia de la velocidad de cizalla sobre la distribución de tamaños de gotas de aceite para una mayonesa estabilizada con proteínas de huevo, a $25^{\circ} \mathrm{C}\left(3 a: 0 \mathrm{~s}^{-1} ; 3 b: 300 \mathrm{~s}^{-1} ; 3 \mathrm{c}: 3000 \mathrm{~s}^{-1}\right)$. 
Consecuentemente, esto parece indicar que ciertos procesos inducidos por la cizalla distintos al de defloculación de las gotas de aceite, limitan la aplicabilidad del modelo de Wagner.

\section{AGRADECIMIENTO}

Este trabajo forma parte de un proyecto de investigación conjunto entre los Departamentos de Ingeniería Química de Cambridge (Reino Unido) y Sevilla (España), subvencionado a través de las Acciones Integradas Hispano-Británicas. Los autores agradecen la concesión de dicha ayuda.

\section{BIBLIOGRAFÍA}

Barnes, H. A. (1995). - «A review of the slip (wall depletion) of polymer solutions, emulsions and particle suspensions in viscometers: its cause, character and cure".- J. NonNewtonian Fluid Mech. 56, 221-251.

Berjano, M., Muñoz, J., Guerrero,. A., Flores, V., Gallegos, C. (1990). - “Comportamiento viscoelástico dinámico de mayonesas comerciales".- Rev. Agroquim. Tecnol. Aliment. 30, 481-491.

De Rosa, M. E., Winter, H. H. (1994). - "The effect of entanglements on the rheological behavior of polybutadiene critical gels".- Rheol. Acta 33, 220-237.

Dickinson, E. (1989). - «Food colloids-An overview».Colloids Surf. 42, 191-204.

Franco. J. M., Guerrero, A., Gallegos, C. (1995). -«Rheology and processing of salad dressing emulsions".- Rheol. Acta 34, 513-524.

Franco, J. M., Berjano, M., Gallegos, C. (1997). -«Linear viscoelasticity of salad dressing emulsions".- J. Agric. Food Chem. 45, 713-719.

Gallegos, C., Berjano, M., García, F. P., Muñoz, J., Flores, V. (1988). - «Aplicación de un modelo cinético al estudio del flujo transitorio en mayonesas".- Grasa y Aceites 39, 254-263.
Gallegos, C., Berjano M., Choplin, L. (1992a). -«Linear viscoelastic behavior of commercial and model mayonnaise".- J. Rheol. 36, 465-478

Gallegos, C., Berjano, M., Guerrero, A., Muñoz, J., Flores, V. (1992b). - «Transient flow of mayonnaise described by a nonlinear viscoelasticity model'.- J. Texture Stud. 23, 153-168.

Gallegos, C., Franco, J. M. (1995).-«Linear and non-linear viscoelasticity of food emulsions containing a mixture of two emulsifiers".- Les Cahiers de Rhéologie 14, 107116.

Larson, R. G. (1988). - "Constitutive equations for polymer melts and solutions".- Butterworths, Boston.

Mackely, M. R., Marshall, R. T. J., Smeulders, J. B. A. F., Zhao, F. D. (1994). - «The rheological characterization of polymeric and colloidal fluids".- Chem. Eng. Sci. 16, 2551-2565.

Madiedo, J. M. (1996). -«Caracterización reológica de emulsiones mediante espectros de relajación y retardación”.Tesis Doctoral de la Universidad de Sevilla, Sevilla.

Madiedo, J. M., Muñoz, J., Gallegos, C. (1996). - «Calculation of relaxation and retardation spectra using the Tikhonov regularization method: application to emulsions" en "Rheology and Fluid Mechanics of Nonlinear Materials» Vol. 217, p. 151.- Siginer D. A. and Advani S. G. (eds.), ASME, New York.

Madiedo, J. M., Gallegos, C. (1997). - «A comparison of methods for the calculation of relaxation and retardation spectra: application to the viscoelastic characterisation of oil-in-water food emulsions".- Food Sci. Technol. Intern. (enviado para publicación).

Melik, D. H., Fogler, H. S. (1988). - «Encyclopedia of Emulsion Technology, II»».- Becher, P. (ed.), Marcel Dekker, New York.

Tadros, Th. F. (1990). - «Use of viscoelastic measurements in studying interactions in concentrated dispersions".Langmuir 6, 28-35.

Tadros., Th. F. (1993). - «Fundamental principles of emulsion rheology and their applications"; en First World Congress on Emulsion, vol. 4, p. 237.- París.

Wagner, M. H. (1976). -«Analysis of time-dependent nonlinear stress growth data for shear and elongational flow of a low-density branched polyethylene melt».- Rheol. Acta 15, 136-142.

Recibido: Mayo 1997 Aceptado: Noviembre 1997 